\title{
Asymptotic efficiency and small sample power of a locally most powerful linear rank test for the log-logistic distribution
}

\author{
Hidetoshi Murakami
}

Received: 5 September 2014/ Accepted: 19 November 2014/Published online: 3 December 2014

(C) The Author(s) 2014. This article is published with open access at Springerlink.com

\begin{abstract}
Assume that two independent random samples are distributed according to a log-logistic distribution (LLD). In this study, the score functions for the locally most powerful rank test were derived for the location and scale parameters. The Wilcoxon rank-sum test was shown to be locally most powerful rank test for the LLD. The asymptotic efficiency of the Wilcoxon rank-sum test was derived and compared with that of the modified Wilcoxon rank-sum test for the LLD.
\end{abstract}

Keywords Asymptotic efficiency - Locally most powerful rank test $\cdot$ Log-logistic distribution - Modified Wilcoxon rank sum test . Wilcoxon rank sum test

\section{Introduction}

Testing hypotheses is one of the most important problems in performing nonparametric statistics. Various nonparametric statistics have been shown to be a locally most powerful rank test (LMPRT) for a specific distribution over the course of many years. For example, [9] obtained the LMPRTs for comparing two possibly censored samples for a given alternative, by deriving scores for censored and uncensored observations. In finance literature, the Lévy distribution arises as a special case for describing security price returns by mixtures of distributions. Runde [13] derived the score functions of LMPRTs for the location and scale parameters with the Lévy distribution. Hájek et al. [5] discussed the LMPRTs for various distributions. The most powerful test

H. Murakami $(\bowtie)$

Department of Mathematics, National Defense Academy, 1-10-20 Hashirimizu, Yokosuka, Kanagawa 239-8686, Japan e-mail: murakami@gug.math.chuo-u.ac.jp for correlation is well-known, and the LMPRT uses FisherYates expected normal scores. However, the bivariate normal distribution does not fit some types of data. Conover [2] considered the LMPRTs for correlation with four examples. Pandit [11] investigated the LMPRT for testing independence against a weighted contamination alternative. Few studies have discussed the LMPRT when only a fraction of treated subjects respond to treatment; however, [12] examined the LMPRT for several of these case types.

Let $\left\{X_{i j} ; i=1,2, j=1, \ldots, n_{i}\right\}$ be two random samples of size $n_{1}$ and $n_{2}$ independent observations, each of which have a continuous distribution described as $F_{1}$ and $F_{2}$, respectively. Assume that $X_{i j}$ is distributed according to a log-logistic distribution (LLD); see e.g., [14], with the probability density function (pdf) $f(x)$ and the cumulative distribution function (cdf) $F(x)$ as follows:

$\begin{aligned} f_{i}(x) & =\frac{\beta_{i}\left\{\left(x-\mu_{i}\right) / \alpha_{i}\right\}^{\beta_{i}-1}}{\alpha_{i}\left[1+\left\{\left(x-\mu_{i}\right) / \alpha_{i}\right\}^{\beta_{i}}\right]^{2}}, \\ F_{i}(x) & =\frac{\left(x-\mu_{i}\right)^{\beta}}{\alpha^{\beta}+\left(x-\mu_{i}\right)^{\beta}}, \quad \alpha_{i}, \beta_{i}>0, \quad x>\mu_{i},\end{aligned}$

respectively. The parameters $\mu, \alpha$ and $\beta$ denote the location, scale and shape parameters, respectively. Note that the median of the LLD is $\alpha+\mu$. This distribution is a special case of Burr's type-XII distribution; see e.g., [1]. In addition, the LLD is a special case of the kappa distributions introduced by [6]. The LLD is often applied to economics as a simple model for wealth or income distribution [3]. Note that the LLD is known as the Fisk distribution in economics. Additionally, the LLD is used in survival analysis as a parametric model for events whose hazard rate increases initially and decreases later.

On the basis of these data types, we proposed the following hypothesis: 


$$
\begin{gathered}
H_{0}: F_{1}(x)=F_{2}(x) \text { against } H_{L}: F_{1}(x)=F_{2}(x-\mu), \\
\mu \neq 0
\end{gathered}
$$

or

$$
\begin{gathered}
H_{0}: F_{1}(x)=F_{2}(x) \quad \text { against } H_{S}: F_{1}(x)=F_{2}(x / \alpha), \\
\alpha>0, \alpha \neq 1 .
\end{gathered}
$$

For a recent comparison study of many nonparametric tests for scale, see [8]. To test these hypotheses, we developed a linear rank statistic. Let $V_{j}=0$ if the $j$ th smallest of the $N=$ $n_{1}+n_{2}$ observations is from $X_{1 j}$; otherwise, $V_{j}=1$. This provides a general two-sample linear rank statistic, as follows:

$T=\sum_{j=1}^{N} a(j) V_{j}=\sum_{j=1}^{n_{2}} a\left(R_{j}\right)$.

Herein, $R_{j}$ denotes the rank of sample $X_{2 j}$. In Sect. 2, we derive a LMPRT for $H_{L}$ and $H_{S}$. Since finite sample sizes are used in practice, we investigate a small sample power of the linear rank tests for the LLD in Sect. 3. Finally, conclusions are presented in Sect. 4.

\section{Locally most powerful linear rank test}

In this section, we derive the LMPRT for $H_{L}$ and $H_{S}$. Assume that $f$ is absolutely continuous and that

$$
\int\left|f^{\prime}(x)\right| d x<\infty
$$

The LMPRT for $H_{L}$ is given by [5], as follows:

$$
\begin{aligned}
T_{L} & =\sum_{j=1}^{N} a_{N}(j, f) V_{j}=\sum_{j=1}^{N} \mathrm{E}\left\{\psi_{L}\left(U_{(j: N)}, f\right)\right\} V_{j} \\
& =\sum_{j=1}^{N} \mathrm{E}\left\{-\frac{f^{\prime}\left(F^{-1}\left(U_{(j: N)}\right)\right)}{f\left(F^{-1}\left(U_{(j: N)}\right)\right)}\right\} V_{j},
\end{aligned}
$$

where $U_{(1: N)}<\cdots<U_{(N: N)}$ are ordered statistics from the uniform distribution in the interval $[0,1]$. In addition, the LMPRT for $H_{S}$ is given by [5] as

$$
\begin{aligned}
T_{S} & =\sum_{j=1}^{N} a_{N}(j, f) V_{j}=\sum_{j=1}^{N} \mathrm{E}\left\{\psi_{S}\left(U_{(j: N)}, f\right)\right\} V_{j} \\
& =\sum_{j=1}^{N} \mathrm{E}\left\{-1-F^{-1}\left(U_{(j: N)}\right) \frac{f^{\prime}\left(F^{-1}\left(U_{(j: N)}\right)\right)}{f\left(F^{-1}\left(U_{(j: N)}\right)\right)}\right\} V_{j} .
\end{aligned}
$$

By Theorem 3.1.2.4 in [5], the score function $a_{N}(j, f)$ can be rewritten as

$$
a_{N}(j, f)=N\left(\begin{array}{c}
N-1 \\
j-1
\end{array}\right) \int_{0}^{1} \psi(u, f) u^{j-1}(1-u)^{N-j} \mathrm{~d} u
$$

LMPRT for the location parameter

In this section, the score function of the LMPRT is derived for the LLD for the location parameter. The standard LLD is given as follows:

$f(x)=\frac{1}{(1+x)^{2}} \quad$ and $\quad F(x)=\frac{x}{1+x}$.

From (2), the first derivative of pdf and the inverse of cdf for the standard LLD are, respectively, given by

$f^{\prime}(x)=-\frac{2}{(1+x)^{3}} \quad$ and $\quad F^{-1}(x)=\frac{x}{1-x}$.

The score function $\psi_{L}\left(U_{(j: N)}, f\right)$ for $H_{L}$ is obtained from the following:

$\psi_{L}\left(U_{(j: N)}, f\right)=2\left(1-U_{(j: N)}\right)$,

and

$\sum_{j=1}^{N} a_{N}\left(U_{(j: N)}, f\right) V_{j}=\frac{2}{N+1} \sum_{j=1}^{n_{2}}\left(N+1-R_{j}\right)$

using (1). (4) denotes the inverse function of $R_{j}$. Thus, the Wilcoxon rank-sum test is the LMPRT for $H_{L}$ with LLDs.

Note that the LMPRT is valid in the neighborhood close to the null hypothesis. In many cases, the asymptotic efficiency of the LMPRT is the highest for the adjusted distribution. However, moderate to large sample sizes are required to assume a specific distribution for deriving the LMPRT. Herein, we consider the asymptotic efficiency of both the original and modified Wilcoxon rank-sum tests for the LLD. Tamura [15] proposed the modified Wilcoxon rank-sum test to raise the asymptotic relative efficiency as follows:

$T(p)=\sum_{i=1}^{N} i^{p} V_{i}=\sum_{i=1}^{n_{2}} R_{i}^{p}, \quad p \in \mathbb{R}^{+}$.

Note that the test statistic $T(p)$ is the original Wilcoxon rank-sum test when $p=1$. By applying the idea of [15] to the score function (3), we can obtain the score function of the inverse rank test:

$\psi_{L}^{*}\left(U_{(j: N)}, f\right)=(p+1)\left(1-U_{(j: N)}\right)^{p}$.

The asymptotic efficiency of the score function (6) for the location parameter is given as follows:

Table 1 Asymptotic efficiency for the location parameter with the LLD

\begin{tabular}{llllllll}
\hline$p$ & 0.1 & 0.5 & 1 & 1.5 & 2 & 2.5 & 3 \\
\hline$A E L(p, f)$ & 0.250 & 0.720 & 1.333 & 2.041 & 2.813 & 3.630 & 4.480 \\
$A R E(p, 1, f)$ & 0.188 & 0.540 & 1.000 & 1.531 & 2.110 & 2.723 & 3.361 \\
\hline
\end{tabular}




$$
\begin{aligned}
\operatorname{AEL}(p, f)= & \lambda(1-\lambda)(2 p+1)(p+1)^{3} \\
& \left(\int_{-\infty}^{\infty} f(x)^{2}\{1-F(x)\}^{p-1} \mathrm{~d} x\right)^{2},
\end{aligned}
$$

where $\lambda=\lim _{N \rightarrow \infty} \lambda_{N}=\lim _{N \rightarrow \infty} n_{1} / N$. The asymptotic efficiency $A E L(p, f)$ and asymptotic relative efficiency $A R E(p, 1, f)$ to the original Wilcoxon rank-sum test for the LLD are listed in Table 1.

The results shown in Table 1 indicate that the asymptotic efficiency of the $T(p)$ test is higher than that of the original Wilcoxon rank-sum test for LLDs when $p>1$.

LMPRT for the scale parameter

Consider the score function of the LMPRT for $H_{S}$. Using a similar procedure as that applied to the location case, we obtain the score function, given below:

$\psi_{S}\left(U_{(i: N)}, f\right)=2 U_{(i: N)}-1$.

Therefore,

$\sum_{j=1}^{N} a_{N}\left(U_{(j: N)}, f\right) V_{j}=\frac{2}{N+1}\left(\sum_{j=1}^{n_{2}} R_{i}-\frac{n_{2}(N+1)}{2}\right)$

using (1). Thus, (7) reveals that the Wilcoxon rank-sum test is the LMPRT for $H_{S}$ with a LLD. Note that the score function of the modified Wilcoxon rank-sum test is given as [4]:

$\psi_{S}^{*}\left(U_{(i: N)}, f\right)=(p+1) U_{(i: N)}^{p}-1$.

From this, the asymptotic efficiency of the modified Wilcoxon rank-sum test for the scale parameter is given by

$$
\begin{aligned}
\operatorname{AES}(p, f)= & \lambda(1-\lambda)(2 p+1)(p+1)^{2} \\
& \times\left(\int_{-\infty}^{\infty} x f(x)^{2} F(x)^{p-1} d x\right)^{2} .
\end{aligned}
$$

The asymptotic efficiency $A E S(p, f)$ and the asymptotic relative efficiency $\operatorname{ARE}(p, 1, f)$ to the original Wilcoxon rank-sum test for the LLD are listed in Table 2.
Therefore, the results shown in Table 2 reveal that the original Wilcoxon rank-sum test is suitable for testing LLDs.

Herein, we consider another distribution, as follows:

$$
\begin{aligned}
& f^{*}(x)=\frac{2^{q}-1}{x^{q+1}\left\{1+\left(2^{q}-1\right) x^{-q}\right\}^{(1+q) / q}}, \\
& F^{*}(x)=\frac{1}{\left\{1+\left(2^{q}-1\right) x^{-q}\right\}^{1 / q}}, \quad(x>0) .
\end{aligned}
$$

For the case $q=1$, this distribution is equivalent to that of the standard LLD. Thus, $f^{*}$ and $F^{*}$ is another extension of the standard LLD. Using a procedure similar to the one presented earlier in this section, the score function $\psi_{S}\left(U_{(i: N)}, f\right)$ is given by

$\psi_{S}\left(U_{(i: N)}, f^{*}\right)=(q+1) U_{(i: N)}^{q}-1$.

Therefore, the modified Wilcoxon rank-sum test is the LMPRT for $H_{S}$ with $f^{*}$. Additionally, we compared the asymptotic efficiency of the modified Wilcoxon rank-sum test with that of the original Wilcoxon rank-sum test for $f^{*}$ in Table 3.

Table 3 shows that the modified Wilcoxon rank-sum test is more efficient than is the original Wilcoxon rank-sum test for $f^{*}$.

\section{Powers of small sample sizes}

In this section, we present our investigation of the behavior of the original Wilcoxon rank-sum test and the modified Wilcoxon rank-sum test for the LLD $f(x)$. In a previous section, we obtained asymptotic results. However, because finite sample sizes are used in practice, we investigated a small sample power of the original and modified Wilcoxon rank-sum tests. Generally, the location, scale, locationscale, and shape parameters of the $X_{1 j}$ and $X_{2 j}$ samples are unequal. Here, we considered a two-sample problem in which the hypothesis, $H_{0}: F_{1}(x)=F_{2}(x)$, was tested against $H_{1}: F_{1}(x) \neq F_{2}(x)$. The following assumptions were made for LLDs, as follows:
Table 2 Asymptotic efficiency for the scale parameter with the LLD

Table 3 Asymptotic efficiency for the scale parameter with the $f^{*}$

\begin{tabular}{llllllll}
\hline$p$ & 0.1 & 0.5 & 1 & 1.5 & 2 & 2.5 & 3 \\
\hline $\operatorname{AES}(p, f)$ & 0.272 & 0.320 & 0.333 & 0.327 & 0.313 & 0.296 & 0.280 \\
$\operatorname{ARE}(p, 1, f)$ & 0.817 & 0.961 & 1.000 & 0.982 & 0.940 & 0.889 & 0.841 \\
\hline
\end{tabular}

\begin{tabular}{llllllll}
\hline$q$ & 0.1 & 0.5 & 1 & 1.5 & 2 & 2.5 & 3 \\
\hline $\operatorname{AES}\left(q, f^{*}\right)$ & 0.008 & 0.125 & 0.333 & 0.562 & 0.800 & 1.042 & 1.286 \\
$\operatorname{AES}\left(1, f^{*}\right)$ & 0.007 & 0.120 & 0.333 & 0.551 & 0.750 & 0.926 & 1.080 \\
$\operatorname{ARE}(q, 1, f)$ & 1.225 & 1.042 & 1.000 & 1.021 & 1.067 & 1.125 & 1.190 \\
\hline
\end{tabular}


Table 4 Simulated power: $n_{1}=n_{2}=10$ for $\alpha=0.05$

\begin{tabular}{|c|c|c|c|c|}
\hline \multirow[t]{2}{*}{$\mu_{2}$} & \multicolumn{4}{|l|}{$\alpha_{2}$} \\
\hline & 1.0 & 2.0 & 3.0 & 4.0 \\
\hline \multicolumn{5}{|c|}{$\operatorname{LLD}(0,1,1)$ and $\operatorname{LLD}\left(\mu_{2}, \alpha_{2}, 1\right)$} \\
\hline \multicolumn{5}{|l|}{0.0} \\
\hline $\mathrm{T}(0.5)$ & 0.044 & 0.312 & 0.748 & 0.959 \\
\hline $\mathrm{T}(0.75)$ & 0.044 & 0.312 & 0.757 & 0.964 \\
\hline $\mathrm{T}(1)$ & 0.044 & 0.318 & 0.761 & 0.965 \\
\hline $\mathrm{T}(2)$ & 0.044 & 0.305 & 0.736 & 0.954 \\
\hline \multicolumn{5}{|l|}{0.5} \\
\hline $\mathrm{T}(0.5)$ & 0.115 & 0.438 & 0.819 & 0.973 \\
\hline $\mathrm{T}(0.75)$ & 0.106 & 0.426 & 0.816 & 0.974 \\
\hline $\mathrm{T}(1)$ & 0.097 & 0.413 & 0.809 & 0.973 \\
\hline $\mathrm{T}(2)$ & 0.074 & 0.360 & 0.764 & 0.958 \\
\hline \multicolumn{5}{|l|}{1.0} \\
\hline $\mathrm{T}(0.5)$ & 0.209 & 0.545 & 0.864 & 0.981 \\
\hline $\mathrm{T}(0.75)$ & 0.187 & 0.522 & 0.855 & 0.981 \\
\hline $\mathrm{T}(1)$ & 0.167 & 0.499 & 0.845 & 0.979 \\
\hline $\mathrm{T}(2)$ & 0.113 & 0.414 & 0.788 & 0.962 \\
\hline \multicolumn{5}{|l|}{1.5} \\
\hline $\mathrm{T}(0.5)$ & 0.310 & 0.628 & 0.895 & 0.986 \\
\hline $\mathrm{T}(0.75)$ & 0.276 & 0.599 & 0.885 & 0.985 \\
\hline $\mathrm{T}(1)$ & 0.244 & 0.570 & 0.872 & 0.983 \\
\hline $\mathrm{T}(2)$ & 0.158 & 0.463 & 0.810 & 0.966 \\
\hline \multicolumn{5}{|c|}{$\operatorname{LLD}(0,1,1)$ and $\operatorname{LLD}\left(\mu_{2}, \alpha_{2}, 0.5\right)$} \\
\hline \multicolumn{5}{|c|}{0.0} \\
\hline $\mathrm{T}(0.5)$ & 0.070 & 0.522 & 0.933 & 0.997 \\
\hline $\mathrm{T}(0.75)$ & 0.059 & 0.489 & 0.918 & 0.996 \\
\hline $\mathrm{T}(1)$ & 0.050 & 0.450 & 0.900 & 0.994 \\
\hline $\mathrm{T}(2)$ & 0.027 & 0.322 & 0.802 & 0.976 \\
\hline \multicolumn{5}{|l|}{0.5} \\
\hline $\mathrm{T}(0.5)$ & 0.142 & 0.599 & 0.944 & 0.998 \\
\hline $\mathrm{T}(0.75)$ & 0.118 & 0.557 & 0.929 & 0.996 \\
\hline $\mathrm{T}(1)$ & 0.098 & 0.515 & 0.911 & 0.994 \\
\hline $\mathrm{T}(2)$ & 0.050 & 0.367 & 0.813 & 0.974 \\
\hline \multicolumn{5}{|l|}{1.0} \\
\hline $\mathrm{T}(0.5)$ & 0.227 & 0.662 & 0.952 & 0.998 \\
\hline $\mathrm{T}(0.75)$ & 0.189 & 0.616 & 0.938 & 0.996 \\
\hline $\mathrm{T}(1)$ & 0.158 & 0.571 & 0.921 & 0.995 \\
\hline $\mathrm{T}(2)$ & 0.080 & 0.408 & 0.825 & 0.975 \\
\hline \multicolumn{5}{|l|}{1.5} \\
\hline $\mathrm{T}(0.5)$ & 0.313 & 0.711 & 0.958 & 0.998 \\
\hline $\mathrm{T}(0.75)$ & 0.264 & 0.664 & 0.944 & 0.997 \\
\hline $\mathrm{T}(1)$ & 0.223 & 0.618 & 0.928 & 0.995 \\
\hline $\mathrm{T}(2)$ & 0.116 & 0.445 & 0.834 & 0.975 \\
\hline
\end{tabular}


Table 4 continued

\begin{tabular}{|c|c|c|c|c|}
\hline \multirow[t]{2}{*}{$\mu_{2}$} & \multicolumn{4}{|l|}{$\alpha_{2}$} \\
\hline & 1.0 & 2.0 & 3.0 & 4.0 \\
\hline \multicolumn{5}{|c|}{$\operatorname{LLD}(0,1,1)$ and $\operatorname{LLD}\left(\mu, \alpha_{2}, 1.5\right)$} \\
\hline \multicolumn{5}{|l|}{0.0} \\
\hline $\mathrm{T}(0.5)$ & 0.036 & 0.195 & 0.517 & 0.813 \\
\hline $\mathrm{T}(0.75)$ & 0.041 & 0.217 & 0.554 & 0.844 \\
\hline $\mathrm{T}(1)$ & 0.046 & 0.236 & 0.584 & 0.865 \\
\hline $\mathrm{T}(2)$ & 0.066 & 0.289 & 0.645 & 0.897 \\
\hline \multicolumn{5}{|l|}{0.5} \\
\hline $\mathrm{T}(0.5)$ & 0.105 & 0.332 & 0.652 & 0.884 \\
\hline $\mathrm{T}(0.75)$ & 0.103 & 0.337 & 0.666 & 0.896 \\
\hline $\mathrm{T}(1)$ & 0.102 & 0.341 & 0.676 & 0.904 \\
\hline $\mathrm{T}(2)$ & 0.102 & 0.348 & 0.689 & 0.913 \\
\hline \multicolumn{5}{|l|}{1.0} \\
\hline $\mathrm{T}(0.5)$ & 0.206 & 0.460 & 0.747 & 0.923 \\
\hline $\mathrm{T}(0.75)$ & 0.192 & 0.451 & 0.747 & 0.927 \\
\hline $\mathrm{T}(1)$ & 0.180 & 0.441 & 0.747 & 0.929 \\
\hline $\mathrm{T}(2)$ & 0.147 & 0.409 & 0.730 & 0.925 \\
\hline \multicolumn{5}{|l|}{1.5} \\
\hline $\mathrm{T}(0.5)$ & 0.315 & 0.567 & 0.811 & 0.946 \\
\hline $\mathrm{T}(0.75)$ & 0.289 & 0.548 & 0.804 & 0.947 \\
\hline $\mathrm{T}(1)$ & 0.266 & 0.530 & 0.798 & 0.946 \\
\hline $\mathrm{T}(2)$ & 0.200 & 0.467 & 0.762 & 0.936 \\
\hline
\end{tabular}

Table 5 Simulated power: $n_{1}=5, n_{2}=10$ for $\alpha=0.05$

\begin{tabular}{|c|c|c|c|c|}
\hline \multirow[t]{2}{*}{$\mu_{2}$} & \multicolumn{4}{|l|}{$\alpha_{2}$} \\
\hline & 1.0 & 2.0 & 3.0 & 4.0 \\
\hline \multicolumn{5}{|c|}{$\operatorname{LLD}(0,1,1)$ and $\operatorname{LLD}\left(\mu_{2}, \alpha_{2}, 1\right)$} \\
\hline \multicolumn{5}{|l|}{0.0} \\
\hline $\mathrm{T}(0.5)$ & 0.050 & 0.253 & 0.610 & 0.878 \\
\hline $\mathrm{T}(0.75)$ & 0.050 & 0.255 & 0.614 & 0.881 \\
\hline $\mathrm{T}(1)$ & 0.050 & 0.255 & 0.612 & 0.876 \\
\hline $\mathrm{T}(2)$ & 0.049 & 0.241 & 0.573 & 0.833 \\
\hline \multicolumn{5}{|l|}{0.5} \\
\hline $\mathrm{T}(0.5)$ & 0.121 & 0.351 & 0.676 & 0.900 \\
\hline $\mathrm{T}(0.75)$ & 0.113 & 0.343 & 0.671 & 0.899 \\
\hline $\mathrm{T}(1)$ & 0.104 & 0.329 & 0.659 & 0.892 \\
\hline $\mathrm{T}(2)$ & 0.080 & 0.285 & 0.599 & 0.840 \\
\hline \multicolumn{5}{|l|}{1.0} \\
\hline $\mathrm{T}(0.5)$ & 0.201 & 0.434 & 0.724 & 0.915 \\
\hline $\mathrm{T}(0.75)$ & 0.184 & 0.417 & 0.714 & 0.912 \\
\hline $\mathrm{T}(1)$ & 0.166 & 0.395 & 0.696 & 0.903 \\
\hline $\mathrm{T}(2)$ & 0.115 & 0.324 & 0.620 & 0.846 \\
\hline \multicolumn{5}{|l|}{1.5} \\
\hline $\mathrm{T}(0.5)$ & 0.276 & 0.502 & 0.760 & 0.926 \\
\hline $\mathrm{T}(0.75)$ & 0.253 & 0.480 & 0.747 & 0.921 \\
\hline $\mathrm{T}(1)$ & 0.228 & 0.452 & 0.726 & 0.912 \\
\hline $\mathrm{T}(2)$ & 0.149 & 0.358 & 0.639 & 0.852 \\
\hline
\end{tabular}


Table 5 continued

\begin{tabular}{|c|c|c|c|c|}
\hline \multirow[t]{2}{*}{$\mu_{2}$} & \multicolumn{4}{|l|}{$\alpha_{2}$} \\
\hline & 1.0 & 2.0 & 3.0 & 4.0 \\
\hline \multicolumn{5}{|c|}{$\operatorname{LLD}(0,1,1)$ and $\operatorname{LLD}\left(\mu_{2}, \alpha_{2}, 0.5\right)$} \\
\hline \multicolumn{5}{|c|}{0.0} \\
\hline $\mathrm{T}(0.5)$ & 0.092 & 0.428 & 0.813 & 0.965 \\
\hline $\mathrm{T}(0.75)$ & 0.084 & 0.407 & 0.795 & 0.959 \\
\hline $\mathrm{T}(1)$ & 0.073 & 0.376 & 0.766 & 0.948 \\
\hline $\mathrm{T}(2)$ & 0.048 & 0.273 & 0.620 & 0.847 \\
\hline \multicolumn{5}{|l|}{0.5} \\
\hline $\mathrm{T}(0.5)$ & 0.160 & 0.487 & 0.829 & 0.967 \\
\hline $\mathrm{T}(0.75)$ & 0.145 & 0.462 & 0.811 & 0.961 \\
\hline $\mathrm{T}(1)$ & 0.126 & 0.427 & 0.782 & 0.951 \\
\hline $\mathrm{T}(2)$ & 0.073 & 0.300 & 0.630 & 0.849 \\
\hline \multicolumn{5}{|l|}{1.0} \\
\hline $\mathrm{T}(0.5)$ & 0.228 & 0.537 & 0.842 & 0.969 \\
\hline $\mathrm{T}(0.75)$ & 0.208 & 0.510 & 0.724 & 0.963 \\
\hline $\mathrm{T}(1)$ & 0.183 & 0.472 & 0.796 & 0.953 \\
\hline $\mathrm{T}(2)$ & 0.100 & 0.325 & 0.638 & 0.850 \\
\hline \multicolumn{5}{|l|}{1.5} \\
\hline $\mathrm{T}(0.5)$ & 0.292 & 0.579 & 0.853 & 0.970 \\
\hline $\mathrm{T}(0.75)$ & 0.269 & 0.551 & 0.835 & 0.964 \\
\hline $\mathrm{T}(1)$ & 0.241 & 0.512 & 0.807 & 0.954 \\
\hline $\mathrm{T}(2)$ & 0.125 & 0.348 & 0.645 & 0.851 \\
\hline \multicolumn{5}{|c|}{$\operatorname{LLD}(0,1,1)$ and $\operatorname{LLD}\left(\mu, \alpha_{2}, 1.5\right)$} \\
\hline \multicolumn{5}{|l|}{0.0} \\
\hline $\mathrm{T}(0.5)$ & 0.033 & 0.154 & 0.406 & 0.693 \\
\hline $\mathrm{T}(0.75)$ & 0.036 & 0.166 & 0.429 & 0.718 \\
\hline $\mathrm{T}(1)$ & 0.040 & 0.178 & 0.451 & 0.738 \\
\hline $\mathrm{T}(2)$ & 0.052 & 0.207 & 0.485 & 0.757 \\
\hline \multicolumn{5}{|l|}{0.5} \\
\hline $\mathrm{T}(0.5)$ & 0.103 & 0.265 & 0.518 & 0.766 \\
\hline $\mathrm{T}(0.75)$ & 0.099 & 0.266 & 0.527 & 0.779 \\
\hline $\mathrm{T}(1)$ & 0.095 & 0.264 & 0.532 & 0.785 \\
\hline $\mathrm{T}(2)$ & 0.086 & 0.258 & 0.528 & 0.778 \\
\hline \multicolumn{5}{|l|}{1.0} \\
\hline $\mathrm{T}(0.5)$ & 0.190 & 0.365 & 0.602 & 0.813 \\
\hline $\mathrm{T}(0.75)$ & 0.177 & 0.356 & 0.601 & 0.817 \\
\hline $\mathrm{T}(1)$ & 0.162 & 0.344 & 0.596 & 0.818 \\
\hline $\mathrm{T}(2)$ & 0.126 & 0.306 & 0.565 & 0.795 \\
\hline \multicolumn{5}{|l|}{1.5} \\
\hline $\mathrm{T}(0.5)$ & 0.274 & 0.450 & 0.665 & 0.845 \\
\hline $\mathrm{T}(0.75)$ & 0.253 & 0.433 & 0.658 & 0.846 \\
\hline $\mathrm{T}(1)$ & 0.230 & 0.413 & 0.646 & 0.842 \\
\hline $\mathrm{T}(2)$ & 0.166 & 0.349 & 0.596 & 0.809 \\
\hline
\end{tabular}

$\operatorname{LLD}(\mu, \alpha, \beta)$ with location parameter $\mu$, scale parameter $\alpha$ and shape parameter $\beta$.

Table 4 and Table 5 show the analysis results of the power of the $T(p)$ for $\left(n_{1}, n_{2}\right)=(10,10)$ and $(5,10)$. The significance level was $5 \%$, and the simulations were repeated $1,000,000$ times. In this study, we assumed $p=$ $0.5,0.75,1$ and 2 . The exact probability of the original
Wilcoxon rank-sum test $T(1)$ is 0.044 for $n_{1}=n_{2}=10$ under the null hypothesis. Thus, we adopted an exact probability of 0.044 for the $T(p)$ test.

The results indicated that a smaller $p$ in the $T(p)$ test was more powerful than a larger $p$ in the $T(p)$ test for equal and unequal sample sizes, with $\beta \leq 1$ for the shifted location, scale, and location-scale parameters. For the case of $\beta>1$, 
a larger $p$ in the $T(p)$ test was more efficient than a small $p$ in the $T(p)$ test for the changed scale parameter, but not the location and location-scale parameters. The results indicated $T(0.5)$ and $T(0.75)$ tests were suitable for a wide range of LLD. Therefore, a smaller $p$ in the $T(p)$ test is suitable for parameters associated with LLDs.

\section{Conclusion and discussions}

In this paper, we considered the LMPRT for the location and scale parameters with the LLD. We showed that the Wilcoxon rank-sum test is suitable for hypothesis testing with the LLD. In addition, the asymptotic efficiency of the modified Wilcoxon rank-sum test was higher than that of the original Wilcoxon rank-sum test for the location parameter with a LLD. For the scale parameter, the asymptotic efficiency of the original Wilcoxon rank-sum test was highest for the LLD. Additionally, the score function of another extension of the LLD was derived as the modified Wilcoxon rank-sum test. However, because finite sample sizes are used in practice, we investigated a small sample power of the original and modified Wilcoxon rank-sum tests. The results indicated that a smaller $p$ in the $T(p)$ test was suitable for parameters associated with LLDs. Future research directions should consider the twosample location-scale problem-i.e., where two null hypotheses $H_{0}$ are simultaneously tested, see; e.g., [7, 10]-for the LLD.

Acknowledgments The author would like to thank the editor and the referee for their valuable comments and suggestions. The author appreciates that this research is supported by the Grant-in-Aid for Young Scientists B of JSPS, KAKENHI Number 26730025.

Open Access This article is distributed under the terms of the Creative Commons Attribution License which permits any use, distribution, and reproduction in any medium, provided the original author(s) and the source are credited.

\section{References}

1. Burr, I.W.: Cumulative frequency functions. Annal Math. Stat. 13, 215-232 (1942)

2. Conover, W.J.: Some locally most powerful rank tests for correlation. J Modern Appl Stat Methods 1, 19-23 (2002)

3. Fisk, P.R.: The graduation of income distributions. Econometrika 29, 171-185 (1961)

4. Goria, M.N.: Some locally most powerful generalized rank tests. Biometrika 67, 497-500 (1980)

5. Hájek, J., Šidák, Z., Sen, P.K.: Theory of rank tests, 2nd edn. Academic Press, San Diego (1999)

6. Mielke, P.W., Johnson, E.S.: Three-parameter kappa distribution maximum likelihood estimates and likelihood ratio tests. Mon Weather Rev 101, 701-709 (1973)

7. Marozzi, M.: Some notes on the location-scale Cucconi test. J Nonparametric Stat. 21, 629-647 (2009)

8. Marozzi, M.: Levene type tests for the ratio of two scales. J Stat. Comput. Simul 81, 815-826 (2011)

9. Moreau, T., Maccario, J., Lellouch, J., Huber, C.: Weighted log rank statistics for comparing two distributions. Biometrika 79, 195-198 (1992)

10. Murakami, H.: Lepage type statistic based on the modified Baumgartner statistic. Comput. Stat. Data Anal 51, 5061-5067 (2007)

11. Pandit, P.V.: Locally most powerful and other rank tests for independence - with a contaminated weighted alternative. Metrika 64, 379-387 (2006)

12. Rosenbaum, P.R.: Confidence intervals for uncommon but dramatic responses to treatment. Biometrics 63, 1164-1171 (2007)

13. Runde, R.: Locally most powerful two-sample rank tests for Lévy distributions. Stat. Papers 39, 179-188 (1998)

14. Shoukri, M.M., Mian, I.U.M., Tracy, D.S.: Sampling Properties of Estimators of the Log-Logistic Distribution with Application to Canadian Precipitation Data. Can. J. Stat. 16, 223-236 (1988)

15. Tamura, R.: On a modification of certain rank tests. Ann. Math. Stat. 34, 1101-1103 (1963) 